Annales Academiæ Scientiarum Fennicæ

Mathematica

Volumen 36, 2011, 603-619

\title{
ON DEVIATIONS, DEFECTS AND ASYMPTOTIC FUNCTIONS OF MEROMORPHIC FUNCTIONS
}

\author{
Liudmyla Kaluzhynova and Ivan Marchenko \\ Kharkiv National University, Department of Mathematics \\ Svobody sq. 4, Kharkiv, 61077, Ukraine; kaluzhynova@univer.kharkov.ua \\ University of Szczecin, Institute of Mathematics \\ ul. Wielkopolska 15, 70-451 Szczecin, Poland; marchenko@wmf.univ.szczecin.pl
}

\begin{abstract}
The structure of the set of deviations $b(q, f)$ of meromorphic functions with $N(r, f)=o(T(r, f))$ from rational functions is investigated. A sharp estimate for the sum of these quantities is obtained. Also we investigated the structure of the set of deviations $b(q, f)$ of meromorphic functions from polynomials. We applied the obtained results to the study of strong asymptotic values of meromorphic functions.
\end{abstract}

\section{Introduction}

We will use the standard notations of the Nevanlinna theory of the distribution of values of meromorphic functions: $m(r, a, f), N(r, a, f), T(r, f), \delta(a, f)$ (see [26] and [18]). Two main theorems of this theory was obtained by Nevanlinna:

Theorem A. For a meromorphic function $f(z)$ and for a point $a \in \overline{\mathbf{C}}$ the following asymptotic equality holds

$$
m(r, a, f)+N(r, a, f)=T(r, f)+O(1), \quad r \rightarrow \infty .
$$

Theorem B. Let $f(z)$ be a meromorphic function and $\left\{a_{k}\right\}_{k=1}^{q} \in \overline{\mathbf{C}}$ be a finite set. Then the inequality

$$
\sum_{k=1}^{q} m\left(r, a_{k}, f\right) \leq 2 T(r, f)+O(\log (r T(r, f)))
$$

is true for all $r \rightarrow \infty$, possibly except for $r$ in a set of finite linear measure.

Theorems $\mathrm{A}$ and $\mathrm{B}$ imply that for all $a \in \overline{\mathbf{C}}$ we have $0 \leq \delta(a, f) \leq 1$ and $\sum_{a \in \overline{\mathbf{C}}} \delta(a, f) \leq 2$

In 1986 the following extension of the second fundamental theorem for the case of rational functions was shown by Frank and Weissenborn.

Theorem C. [8] Let $f$ be a transcendental meromorphic function. Then for distinct rational functions $q_{1}(z), \ldots, q_{m}(z)$ we have

$$
m(r, f)+\sum_{k=1}^{m} m\left(r, q_{k}, f\right) \leq(2+o(1)) T(r, f)
$$

doi:10.5186/aasfm.2011.3638

2010 Mathematics Subject Classification: Primary 30D35; Secondary 30D30.

Key words: Meromorphic function, deviation, asymptotic function. 
for $r \rightarrow \infty$, possibly except for $r$ in a set of finite linear measure.

Also in 1986 Steinmetz proved a more general result for the case of small meromorphic functions.

Theorem D. [31] Let $f$ be a nonconstant meromorphic function and let $a_{1}(z), \ldots$, $a_{m}(z)$ be a set of pairwise distinct meromorphic functions such that $T\left(r, a_{k}\right)=$ $o(T(r, f))(r \rightarrow \infty, k=1, \ldots, m)$. Then

$$
m(r, f)+\sum_{k=1}^{m} m\left(r, a_{k}, f\right) \leq(2+o(1)) T(r, f)
$$

for $r \rightarrow \infty$, possibly except for $r$ in a set of finite linear measure.

In 1969 Petrenko set up a following question: how will Nevanlinna's theory change if we measure the proximity of a meromorphic function $f$ to a value $a$ applying a different metric? In order to find the answer he introduced the function of deviation

$$
\mathscr{L}(r, a, f)= \begin{cases}\max _{|z|=r} \log ^{+} \frac{1}{|f(z)-a|}, & a \neq \infty, \\ \max _{|z|=r} \log ^{+}|f(z)|, & a=\infty .\end{cases}
$$

The quantity

$$
\beta(a, f)=\liminf _{r \rightarrow \infty} \frac{\mathscr{L}(r, a, f)}{T(r, f)}
$$

is called the magnitude of deviation. It is clear that $\delta(a, f) \leq \beta(a, f)$ for $a \in \overline{\mathbf{C}}$.

In the case of meromorphic functions of finite lower order $\lambda=\liminf _{r \rightarrow \infty} \frac{\log T(r, f)}{\log r}<$ $\infty$, the properties of $\beta(a, f)$ are very similar to properties of $\delta(a, f)$. Petrenko obtained a sharp upper estimate for the value $\beta(a, f)$ and also some estimates for the $\operatorname{sum} \sum_{a \in \overline{\mathbf{C}}} \beta(a, f)$.

Theorem E. [28] If $f(z)$ is a meromorphic function of finite lower order $\lambda$, then for all $a \in \overline{\mathbf{C}}$ we have

$$
\begin{aligned}
\beta(a, f) \leq B(\lambda):= \begin{cases}\frac{\pi \lambda}{\sin \pi \lambda}, & \text { if } \lambda \leq 0.5, \\
\pi \lambda, & \text { if } \lambda>0.5,\end{cases} \\
\sum_{a \in \overline{\mathbf{C}}} \beta(a, f) \leq 816 \pi(\lambda+1)^{2} .
\end{aligned}
$$

The example of entire Mittag-Leffler function

$$
E_{\lambda}(z)=\sum_{n=0}^{\infty} \frac{z^{n}}{\Gamma\left(1+\frac{n}{\lambda}\right)},
$$

where $\Gamma(x)$ is Euler's gamma function, shows that estimates (1.1) and (1.2) are sharp (see $[12])$.

It should be noted that estimate (1.1) was obtained earlier (see [11]). In 1932 Paley [27] stated a hypothesis that the above inequality (1.2) holds for entire function $f(z)$ of finite lower order and $a=\infty$. This statement was proved in 1969 by Govorov [13]. In 1969 Petrenko obtained this estimate in the general case, applying his method based on the new representation of a meromorphic function in a sector. Now this representation is called a Petrenko's formula. 
In 1990 Marchenko and Shcherba presented the exact estimate of the sum of deviations for meromorphic functions of finite lower order, therefore it was solved the problem posed by Petrenko in his monograph [29].

Theorem F. [24] If $f(z)$ is a meromorphic function of finite lower order $\lambda$, then

$$
\sum_{a \in \overline{\mathbf{C}}} \beta(a, f) \leq 2 B(\lambda)
$$

The estimate of the quantity $\beta(a, f)$ by Valiron's deficiency

$$
\Delta(a, f):=\limsup _{r \rightarrow \infty} \frac{m(r, a, f)}{T(r, f)}
$$

was obtained by Shea (see, for example $[29,9]$ ).

Theorem G. If $f(z)$ is a meromorphic function of finite lower order $\lambda$, then for all $a \in \overline{\mathbf{C}}$ we have

$$
\beta(a, f) \leq B(\lambda, \Delta):= \begin{cases}\pi \lambda \sqrt{\Delta(2-\Delta)} & \text { if } \lambda \geq 0.5 \text { or } 0<\lambda<0.5 \\ \pi \lambda\left(\Delta \operatorname{ctg}(\pi \lambda)+\operatorname{tg} \frac{\pi \lambda}{2}\right) & \text { and } \sin \frac{\pi \lambda}{2} \geq \sqrt{\frac{\Delta}{2}}, \\ \Delta & \text { if } 0<\lambda<0.5 \text { and } \sin \frac{\pi \lambda}{2}<\sqrt{\frac{\Delta}{2}},\end{cases}
$$

where

$$
\Delta=\Delta(a, f) .
$$

In 1982 Ryzhkov [30] built an example of a function, which shows that the estimate in Theorem $G$ is exact. Theorem $G$ implies that for a meromorphic function with finite lower order

$$
\Omega(f)=\{a \in \overline{\mathbf{C}}: \beta(a, f)>0\} \subset V(f)=\{a \in \overline{\mathbf{C}}: \Delta(a, f)>0\} .
$$

In 1999 Marchenko obtained the estimate of the sum $\sum_{a \neq \infty} \beta(a, f)$ by Valiron's deficiency $\Delta\left(0, f^{\prime}\right)$.

Theorem H. [21] Let $f(z)$ be a meromorphic function of finite lower order $\lambda$. Then

$$
\sum_{a \neq \infty} \beta(a, f) \leq 2 B\left(\lambda, \Delta\left(0, f^{\prime}\right)\right)
$$

In 2007 Ciechanowicz and Marchenko studied of deviations of entire functions of finite lower order from rational functions. These results imply the following theorem.

Theorem I. [3] Let $f(z)$ be a transcendental entire function of finite lower order $\lambda$ and $\mathfrak{M}$ be a set of all rational functions. Then the set $\{q \in \mathfrak{M}: \beta(q, f)>0\}$ is at most countable and

$$
\sum_{q \in \mathfrak{M}} \beta(q, f) \leq B(\lambda)
$$

For meromorphic functions of infinite lower order the quantity $\beta(a, f)$ can be infinite. Therefore, in this case the result of Bergweiler and Bock from 1994 is especially interesting. 
Theorem J. [2] For a meromorphic function of infinite lower order

$$
\liminf _{r \rightarrow \infty} \frac{\mathscr{L}(r, \infty, f)}{r T_{-}^{\prime}(r, f)} \leq \pi,
$$

where $T_{-}(r, f)$ is the left derivative of the Nevanlinna characteristic function.

Let us notice that $r T_{-}^{\prime}(r, f)=A(r, f)+O(1), r \rightarrow \infty$, where $A(r, f) \pi$ is the spherical area, counting multiplicities of the covering, of the image on the Riemann's sphere of the disk $\{z:|z| \leq r\}$ under $f$.

In connection with above theorem, Eremenko in 1997 introduced the quantity

$$
b(a, f)=\liminf _{r \rightarrow \infty} \frac{\mathscr{L}(r, a, f)}{A(r, f)} .
$$

The theorem of Bergweiler and Bock implies that for each $a \in \overline{\mathbf{C}}: b(a, f) \leq \pi$. In 1997 Eremenko received an analogue of the the defect relation for the magnitude of deviation $b(a, f)$.

Theorem K. [5] For the meromorphic function such that the set $\{a \in \overline{\mathbf{C}}: b(a, f)$ $>0\}$ contains more than one point we have

$$
\sum_{a \in \overline{\mathbf{C}}} b(a, f) \leq 2 \pi
$$

In 1998 Marchenko received a sharp estimate for $b(a, f)$ by Valiron's deficiency $\Delta_{1}=\Delta(a, f)$ and some estimate for the sum of such deviations by Valiron's deficiency $\Delta_{2}=\Delta\left(0, f^{\prime}\right)$.

Theorem L. [20] If $f(z)$ is a meromorphic function of lower order $\lambda: 0<\lambda \leq \infty$, then for all $a \in \overline{\mathbf{C}}$ we have

$$
b(a, f) \leq \begin{cases}\pi \sqrt{\Delta_{1}\left(2-\Delta_{1}\right)} & \text { if } \lambda \geq 0.5 \text { or } 0<\lambda<0.5 \text { and } \sin \frac{\pi \lambda}{2} \geq \sqrt{\frac{\Delta_{1}}{2}} \\ \pi\left(\Delta_{1} \operatorname{ctg}(\pi \lambda)+\operatorname{tg} \frac{\pi \lambda}{2}\right) & \text { if } 0<\lambda<0.5 \text { and } \sin \frac{\pi \lambda}{2}<\sqrt{\frac{\Delta_{1}}{2}}\end{cases}
$$

For the meromorphic function with lower order $0.5 \leq \lambda \leq \infty$

$$
\sum_{a \neq \infty} b(a, f) \leq 2 \pi \sqrt{\Delta_{2}\left(2-\Delta_{2}\right)}
$$

In 2009 the autors investigated the magnitudes of deviations $b(q, f)$ of entire functions of lower order $\lambda>0$ from rational functions.

Theorem M. [17] Let $f(z)$ be an entire functions of lower order $\lambda>0$. Let also $\mathfrak{M}$ be a set of all rational functions. Then the set $\Omega=\{q \in \mathfrak{M}: b(q, f)>0\}$ is at most countable and

$$
\sum_{q \in \mathfrak{M}} b(q, f) \leq \begin{cases}\frac{\pi}{\sin \pi \lambda} & \text { if } \quad 0<\lambda \leq 0.5 \\ \pi & \text { if } \quad 0.5<\lambda \leq \infty\end{cases}
$$

The example of $f(z)=\int_{0}^{z} e^{-t^{q}} d t$ (see [16] p. 78) shows that for an entire function of finite order $q \in \mathbf{N}$ the estimate from Theorem $\mathrm{J}$ is sharp.

Let us remind the definitions of asymptotic value of a meromorphic function (see [12] p. 233). The value $a \in \overline{\mathbf{C}}$ is called an asymptotic value of a meromorphic function 
$f(z)$ if there exists a curve $\Gamma \subset \mathbf{C}$ given by the equation $z=z(t), 0 \leq t<\infty, \quad z(t) \rightarrow$ $\infty(t \rightarrow \infty)$, such that $\lim _{z \rightarrow \infty, z \in \Gamma} f(z)=\lim _{t \rightarrow \infty} f(z(t))=a$. In 1918 Gross [14] built an example of an entire function of infinite order for which the set of asymptotic values coincides with the whole extended complex plane $\overline{\mathbf{C}}$.

A classical theorem of Denjoy-Carleman-Ahlfors gives the sharp upper estimate of the number of asymptotic values in case of entire functions of finite lower order.

Theorem N. [12] An entire function of finite lower order $\lambda$ cannot have more than $[2 \lambda]+1$ different asymptotic values, where $[x]$ is the integer part of $x$.

In case of meromorphic functions, the number of asymptotic values may be infinite even for functions of finite order. The following theorem was proved by Eremenko in 1986.

Theorem O. [4] For every value $\rho, 0 \leq \rho \leq \infty$, there exists a meromorphic function of order $\rho$ with the set of asymptotic values equal to $\overline{\mathbf{C}}$.

In 2004 Marchenko introduced the strong asymptotic values of a meromorphic function. We say that $a \in \overline{\mathbf{C}}$ is an $\alpha_{0}$-strong asymptotic value of a meromorphic function $f$, if the exists a curve $\Gamma \subset \mathbf{C}: z=z(t), 0 \leq t<\infty, z(t) \rightarrow \infty(t \rightarrow \infty)$, such that

$$
\begin{gathered}
\liminf _{t \rightarrow \infty} \frac{\log |f(z(t))-a|^{-1}}{T(|z(t)|, f)}=\alpha(a) \geq \alpha_{0}>0 \quad \text { if } a \neq \infty, \\
\liminf _{t \rightarrow \infty} \frac{\log |f(z(t))|}{T(|z(t)|, f)} \geq \alpha_{0}>0 \quad \text { if } a=\infty .
\end{gathered}
$$

It is easy to notice that if $a$ is a strong asymptotic value of $f$ then the magnitude of Petrenko's deviation $\beta(a, f) \geq \alpha_{0}$. It means that $a$ is also a defective value in the sense of Petrenko.

Theorem P. $[22]^{1}$ Let $f(z)$ be a meromorphic function of finite lower order $\lambda$ and $\left\{a_{\nu}\right\}, \nu=1,2, \ldots, p-\alpha_{0}$-strong asymptotic values of $f(z)$. Then $p \leq\left[\frac{2 B(\lambda)}{\alpha_{0}}\right]$.

In 1907 Denjoy made the following conjecture: if $f(z)$ is an entire function of finite lower order $\lambda$, if also $a_{1}, a_{2}, \ldots, a_{p}$ are an entire functions of order less than $\frac{1}{2}$, such that $f(z)-a_{j}(z) \rightarrow 0$ for $z$ tending to infinity along the path $\Gamma_{j}, 1 \leq j \leq p$, then $p \leq[2 \lambda]$. This inequality has been solved for a case of entire functions $\left(a_{k}(z)\right)$ of order less than $\frac{1}{4}$ by Fenton in 1983 [7]. In the general case the conjecture is still open until now.

If we look at the function $f(z)=e^{z}$ and rational functions $a_{c}(z)=\frac{c}{z}, c \in \mathbf{C}$, we can see, that for each $c, f(z)-a_{c}(z) \rightarrow 0$ for $z$ tending to infinity along the path $\Gamma: z=-t, 0 \leq t<+\infty$. This illustrates the fact, that the number of asymptotic functions can be infinite if the functions considered $\left(a_{c}(z)\right)$ are not entire.

In case $\lambda=\frac{1}{2}$ for the function $f(z)=\frac{\sin \sqrt{z}}{\sqrt{z}}(f(0)=1)$ we have that $b_{c}(z)=c \frac{\sin \sqrt{z}}{\sqrt{z}}$, $c \in \mathbf{C}$, are its asymptotic functions. Indeed, $f(x)-b_{c}(x)=(1-c) \frac{\sin \sqrt{x}}{\sqrt{x}} \rightarrow 0$ for $x \rightarrow+\infty$. The number of those functions is infinite. It is easy to see that these asymptotic functions are not strong asymptotic functions for the function $f(z)$. It

\footnotetext{
${ }^{1}$ It should be mentioned here that in [22] not only the theorem for strong asymptotic values is obtained, but also a more general result for strong asymptotic spots.
} 
would be interesting to consider if Denjoy's hypothesis holds not only for asymptotic functions, but also for strong asymptotic functions of meromorphic functions.

In 2008 Marchenko introduced the notation of an $A$-strong asymptotic function of meromorphic function. Meromorphic function $q(z)$ is called an $A$-strong asymptotic function of meromorphic function $f(z)$ if there exists a curve $\Gamma \subset \mathbf{C}$ given by the equation $z=z(t), 0 \leq t<\infty, z(t) \rightarrow \infty(t \rightarrow \infty)$, such that

$$
\liminf _{t \rightarrow \infty} \frac{\log \frac{1}{|f(z(t))-q(z(t))|}}{A(|z(t)|, f)} \geq \alpha_{0}>0 .
$$

Thus, $q(z)$ is $A$-strong asymptotic function of a meromorphic function $f(z)$, if the rate of convergence to zero of the difference $f(z)-q(z)$ on asymptotic curve is comparable with the growth of the Ahlfors-Shimizu characteristic $(A(r, f))$. We should say that $A(r, f)=\iint_{|z| \leq r} \frac{\left|f^{\prime}(z)\right|^{2}}{\left(1+\left|f^{\prime}(z)\right|^{2}\right)^{2}} d x d y(z=x+i y)$ is double integral over the circle $|z| \leq r$ of the square modulus of the spherical derivative of $f(z)$. This characteristic is an analog of the Dirichlet integral of the holomorphic in the unit disk function $\left(I(r, f)=\iint_{|z| \leq r}\left|f^{\prime}(z)\right|^{2} d x d y, r<1, z=x+i y\right)$ for the meromorphic case.

Theorem Q. [23] Let $f(z)$ be an entire function of infinite lower order. Then the number of A-strong asymptotic rational functions is finite and less or equal to $\left[\frac{\pi}{\alpha_{0}}\right]$, where $[x]$ is the integer part of $x$.

\section{Main results}

Let $\mathfrak{M}$ be a set of all rational functions. Let also $\mathfrak{P}$ be a set of all polynomials.

Theorem 1. Let $f(z)$ be a meromorphic function of lower order $\lambda, 0<\lambda \leq \infty$, with

$$
N(r, \infty, f)=o(T(r, f)) \quad(r \rightarrow \infty)
$$

Then the set $\Omega=\{q \in \mathfrak{M}: b(q, f)>0\}$ is at most countable and

$$
\sum_{q \in \mathfrak{M}} b(q, f) \leq \begin{cases}\frac{\pi}{\sin \pi \lambda} & \text { if } 0<\lambda \leq 0.5 \\ \pi & \text { if } \lambda>0.5\end{cases}
$$

It is easy to notice that if $q(z)$ is a $A$-strong asymptotic rational function of meromorphic function $f$ then the magnitude of Eremenko's deviation $b(q, f) \geq \alpha_{0}$. There from Theorem 1 we have

Corollary 1.1. Let $f(z)$ be a meromorphic function of lower order $\lambda, 0<\lambda \leq \infty$, with (2.1). Then the number $p$ of A-strong asymptotic rational functions is finite and

$$
p \leq \begin{cases}{\left[\frac{\pi}{\alpha_{0} \sin \pi \lambda}\right]} & \text { if } 0<\lambda \leq 0.5 \\ {\left[\frac{\pi}{\alpha_{0}}\right]} & \text { if } \lambda>0.5\end{cases}
$$


Theorem 2. Let $f(z)$ be a meromorphic function of lower order $\lambda, 0<\lambda \leq \infty$, let also $\mathfrak{P}_{d}$ be a set of all polynomials of degree not greater than $d$. Then

$$
\sum_{q \in \mathfrak{P}_{d}} b(q, f) \leq \begin{cases}\pi(d+2) \sqrt{\Delta(2-\Delta)} & \text { if } \lambda \geq 0.5 \text { or } 0<\lambda<0.5 \\ \pi(d+2)\left(\Delta \operatorname{ctg}(\pi \lambda)+\operatorname{tg} \frac{\pi \lambda}{2}\right) & \text { and } \sin \frac{\pi \lambda}{2} \geq \sqrt{\frac{\Delta}{2}}, \\ \text { if } 0<\lambda<0.5 \text { and } \sin \frac{\pi \lambda}{2}<\sqrt{\frac{\Delta}{2}}\end{cases}
$$

where $\Delta=\Delta\left(0, f^{(d+1)}\right)$.

Corollary 2.1. Let $f(z)$ be a meromorphic function of lower order $\lambda, 0<\lambda \leq \infty$, let also $\mathfrak{P}_{d}$ be a set of all polynomials of degree not greater than $d$. Then the set $\left\{q \in \mathfrak{P}_{d}: b(q, f)>0\right\}$ is at most countable and

$$
\sum_{q \in \mathfrak{P}_{d}} b(q, f) \leq \begin{cases}\frac{(2+d) \pi}{\sin \pi \lambda} & \text { if } 0<\lambda \leq 0.5 \\ (2+d) \pi & \text { if } \lambda>0.5\end{cases}
$$

Corollary 2.2. Let $f(z)$ be a meromorphic function of lower order $\lambda, 0<\lambda \leq \infty$. Then the number $q$ of $A$-strong asymptotic polynomials of degree not greater than $d$ is finite and

$$
q \leq \begin{cases}\frac{\pi(d+2)}{\alpha_{0}} \sqrt{\Delta(2-\Delta)} & \text { if } \lambda \geq 0.5 \text { or } 0<\lambda<0.5 \text { and } \sin \frac{\pi \lambda}{2} \geq \sqrt{\frac{\Delta}{2}} \\ \frac{\pi(d+2)}{\alpha_{0}}\left(\Delta \operatorname{ctg}(\pi \lambda)+\operatorname{tg} \frac{\pi \lambda}{2}\right) & \text { if } 0<\lambda<0.5 \text { and } \sin \frac{\pi \lambda}{2}<\sqrt{\frac{\Delta}{2}}\end{cases}
$$

where $\Delta=\Delta\left(0, f^{(d+1)}\right)$.

\section{Auxiliary results}

Bergweiler and Bock in [2] introduced a generalization of Polya peaks to functions of infinite lower order. Let us remind the basic construction.

For all sequences $M_{j} \rightarrow \infty, \varepsilon_{j} \rightarrow 0$ there exit sequences $\rho_{j} \rightarrow \infty$ and $\mu_{j} \rightarrow \infty$ such that, for all $r^{\prime}$ s fulfilling inequality $\left|\log \left(r / \rho_{j}\right)\right| \leq M_{j} / \mu_{j}$, we have

$$
T(r, f) \leq\left(1+\varepsilon_{j}\right)\left(\frac{r}{\rho_{j}}\right)^{\mu_{j}} T\left(\rho_{j}, f\right) \quad(j \rightarrow \infty) .
$$

We can choose the sequences $\mu_{j}$ and $M_{j}$ such that

$$
\mu_{j}=o\left(\log ^{3 / 2} T\left(\rho_{j}, f\right)\right), \quad M_{j}=o\left(\log T\left(\rho_{j}, f\right)\right) \quad(j \rightarrow \infty) .
$$

Let us put

$$
P_{j}=\rho_{j} e^{-M_{j} / \mu_{j}}, \quad Q_{j}=\rho_{j} e^{M_{j} / \mu_{j}} .
$$

Then the inequality (3.1) is true for all $r \in\left[P_{j}, Q_{j}\right]$. We shall assume that $M_{j}>1$.

Let us consider the sets

$$
\begin{aligned}
& A_{j}=\left\{r \in\left[\rho_{j}, Q_{j}\right]: T(r, f) \leq \frac{1}{\sqrt{\mu_{j}}}\left(\frac{r}{\rho_{j}}\right)^{\mu_{j}} T\left(\rho_{j}, f\right)\right\}, \\
& B_{j}=\left\{r \in\left[P_{j}, \rho_{j}\right]: T(r, f) \leq \frac{1}{\sqrt{\mu_{j}}}\left(\frac{r}{\rho_{j}}\right)^{\mu_{j}} T\left(\rho_{j}, f\right)\right\} .
\end{aligned}
$$


Let us put

$$
\begin{aligned}
& R_{j}=\left\{\begin{array}{ll}
\min A_{j} & \text { if } A_{j} \neq \emptyset, \\
Q_{j} & \text { if } A_{j}=\emptyset,
\end{array} t_{j}= \begin{cases}\max B_{j} & \text { if } B_{j} \neq \emptyset, \\
P_{j} & \text { if } B_{j}=\emptyset,\end{cases} \right. \\
& S_{j}=e^{-1 / \mu_{j}} R_{j}, \quad T_{j}=e^{-2 / \mu_{j}} R_{j} .
\end{aligned}
$$

Then

$$
t_{j}<\rho_{j}<T_{j}<S_{j}<R_{j}
$$

In [2] it is shown that

$$
\frac{T\left(R_{j}, f\right)}{R_{j}^{\mu_{j}}}+\frac{T\left(t_{j}, f\right)}{t_{j}^{\mu_{j}}}=o\left(\mu_{j} \int_{t_{j}}^{T_{j}} \frac{T(r, f)}{r^{\mu_{j}+1}} d r\right) \quad(j \rightarrow \infty) .
$$

Apart from that, it follows from the inequality (19) in [2] that

$$
T\left(\rho_{j}, f\right) \leq T^{3 / 2}\left(t_{j}, f\right), \quad j \rightarrow \infty .
$$

The following lemmas are the version of the lemma on logarithmic derivative which has been applied in [17].

Lemma A. [17] Let $f(z)$ be a meromorphic function of infinite lower order. Then for every natural number $k$ we have

$$
\int_{t_{j}}^{T_{j}} \frac{m\left(r, f^{(k)} / f\right)}{r^{\mu_{j}+1}} d r=o\left(\int_{t_{j}}^{T_{j}} \frac{T(r, f)}{r^{\mu_{j}+1}} d r\right), \quad j \rightarrow \infty .
$$

Lemma B. [17] Let $f(z)$ be a meromorphic function of infinite lower order. Then for every natural number $k$ we have

$$
\int_{t_{j}}^{T_{j}} \frac{\mathscr{L}\left(r, f^{(k)} / f\right)}{r^{\mu_{j}+1}} d r=o\left(\mu_{j} \int_{t_{j}}^{T_{j}} \frac{T(r, f)}{r^{\mu_{j}+1}} d r\right), \quad j \rightarrow \infty,
$$

where

$$
\mathscr{L}\left(r, \frac{f^{(k)}}{f}\right)=\log ^{+} \max _{|z|=r}\left|\frac{f^{(k)}(z)}{f(z)}\right| .
$$

Also, we need one more fact from the analysis.

Lemma C. [19] Let $f(z)$ be a nondecreasing function on the interval $[a, b]$, and $\varphi(x)$ be a nonnegative function with bounded derivative on the interval $[a, b]$. Then

$$
\int_{a}^{b} f^{\prime}(x) \varphi(x) d x \leq f(b) \varphi(b)-f(a) \varphi(a)-\int_{a}^{b} \varphi^{\prime}(x) f(x) d x .
$$

For the case of $\varphi(x)=1$ Lemma $\mathrm{C}$ is proved in [25]. 


\section{Proof of Theorem 1}

First we shall prove the statement for meromorphic functions $f(z)$ of infinite lower order and for the case if $\left\{q_{k}\right\}$ are polynomials. Let $\left\{p_{k}(z)\right\}_{k=1}^{m}$ be distinct polynomials. Let also $d=\max \operatorname{deg}\left(p_{k}\right)$ and $b\left(p_{k}, f\right)>0, k=1,2, \ldots, m$. Let the number $t_{0}$ be chosen in such way that for all $|z|>t_{0}$ we have $p_{n}(z) \neq p_{k}(z)(n \neq k)$. We put

$$
c_{k, n}=\min _{|z| \geq t_{0}}\left|p_{n}(z)-p_{k}(z)\right|>0, \quad c=\min _{k, n}\left\{c_{k, n}\right\}>0 .
$$

For $j \geq n_{0}$ we consider the set

$$
G_{j}=\left\{z: t_{j}<|z|<T_{j}, \log \left|f^{(d+1)}(z)\right|<-\sqrt{T\left(\rho_{j}, f\right)}\right\},
$$

where $t_{j}, \rho_{j}, T_{j}$ are sequences from (3.1) and (3.3), and $n_{0}$ is chosen in such way that $t_{n_{0}}>t_{0}$.

Let $G_{j, k}$ be a set consisting of those connected components of $G_{j}$, which contain a points $z_{1}, z_{2}, \ldots, z_{d+1}$ such that

$$
\begin{gathered}
\left|f\left(z_{1}\right)-p_{k}\left(z_{1}\right)\right|<\frac{c}{4}, \\
\left|f^{(l-1)}\left(z_{l}\right)-p_{k}^{(l-1)}\left(z_{l}\right)\right|<\exp \left(-\sqrt{T\left(\rho_{j}, f\right)}\right) \quad(l=2, \ldots, d+2) .
\end{gathered}
$$

In [17] it was shown, that for $j \geq j_{0}$ the sets $G_{j, k}, k=1,2, \ldots, m$, are disjoint.

For $j \geq j_{0}$, let us put

$$
u_{j, k}(z)= \begin{cases}\log \frac{1}{\left|f^{(d+1)}(z)\right|} & \text { if } z \in G_{j, k}, \\ \sqrt{T\left(\rho_{j}, f\right)} & \text { if } z \notin G_{j, k} .\end{cases}
$$

We should note here that each $u_{j, k}(z)(k=1,2, \ldots, m)$ is a $\delta$-subharmonic function in $K\left(t_{j}, T_{j}\right)$.

Let us recall the definition and basic properties of Baernstein's function $T^{*}$. For a complex number $z=r e^{i \theta}, r \in\left(t_{j}, T_{j}\right), \theta \in[0, \pi]$ we put [1]

$$
m^{*}\left(z, u_{j, k}\right)=\sup _{|E|=2 \theta} \frac{1}{2 \pi} \int_{E} u_{j, k}\left(r e^{i \varphi}\right) d \varphi, \quad T^{*}\left(z, u_{j, k}\right)=m^{*}\left(z, u_{j, k}\right)+N\left(r, u_{j, k}\right),
$$

where $|E|$ is Lebesgue's measure of the set $E$ and $N\left(r, u_{j, k}\right)$ counts the zeros of $f^{(d+1)}(z)$ in $G_{j, k} \cap\{z:|z|<r\}$.

Let us put $\widetilde{u}_{j, k}(z)$ for the circular symmetrization of the function $u_{j, k}(z)$ [15]. Let us notice that $\widetilde{u}_{j, k}\left(r e^{i \varphi}\right)$ is a nonnegative, nonincreasing on $[0, \pi]$ even function of $\varphi$, equimeasurable for each fixed $r, 0<r<\infty$ with $u_{j, k}(z)$, and

$$
m^{*}\left(z, u_{j, k}\right)=\frac{1}{\pi} \int_{0}^{\theta} \widetilde{u}_{j, k}\left(r e^{i \varphi}\right) d \varphi
$$

From Baernstein's theorem [1] the function $T^{*}\left(z, u_{j, k}\right)(k=1,2, \ldots, m)$ is subharmonic on

$$
K=\left\{r e^{i \theta}: t_{j}<r<T_{j}, 0<\theta<\pi\right\}
$$


continuous on $K \cup\left(-T_{j},-t_{j}\right) \cup\left(t_{j}, T_{j}\right)$ and logarithmically convex in $r>0$ for each fixed $\theta \in[0, \pi]$. What is more, we have

$$
\begin{aligned}
T^{*}\left(r, u_{j, k}\right) & =N\left(r, u_{j, k}\right), \quad T^{*}\left(r e^{i \pi}, u_{j, k}\right)=T\left(r, u_{j, k}\right), \\
\frac{\partial}{\partial \theta} T^{*}\left(r e^{i \theta}, u\right) & =\frac{\widetilde{u}_{j, k}\left(r e^{i \theta}\right)}{\pi} \quad \text { for } 0<\theta<\pi,
\end{aligned}
$$

where $T\left(r, u_{j, k}\right)$ is the Nevanlinna characteristic of $\delta$-subharmonic function $u_{j, k}(z)$.

Let us put [24]

$$
T_{0}^{*}(z, f)=\sum_{k=1}^{m} T^{*}\left(z, u_{n, k}\right) .
$$

Also for each $\alpha, 0<\alpha<\pi /\left(2 \mu_{j}\right), r \in\left(t_{j}, T_{j}\right)$, we put $[6,10]$

$$
\sigma(r)=\int_{0}^{\alpha} T_{0}^{*}\left(r e^{i \theta}, f\right) \sin \left(\mu_{j}(\alpha-\theta)\right) d \theta .
$$

In [17] (p. 32) the following inequality was obtained

$$
\begin{aligned}
& \int_{t_{j}}^{T_{j}}\left(\frac{1}{\pi} \sum_{k=1}^{m} \widetilde{u}_{j, k}(r, 0) \sin \left(\mu_{j} \alpha\right)-\mu_{j} T_{0}^{*}\left(r e^{i \alpha}, f\right)+\mu_{j} \cos \left(\mu_{j} \alpha\right) N\left(r, 0, f^{(d+1)}\right)\right) \frac{d r}{r^{\mu_{j}+1}} \\
& \leq\left.\left(\frac{\sigma(r)}{r^{\mu_{j}-1}}+\mu_{j} \frac{\sigma(r)}{r^{\mu_{j}}}\right)\right|_{t_{j}} ^{T_{j}}
\end{aligned}
$$

It follows from the definition of function $T_{0}^{*}(z, f),(2.1)$ and $(3.5)$ that for all $\theta \in[0, \pi]$, $r \in\left[t_{j}, T_{j}\right]$ we have

$$
\begin{aligned}
T_{0}^{*}(z, f) & \leq m\left(r, 0, f^{(d+1)}\right)+m \sqrt{T\left(\rho_{j}, f\right)}+N\left(r, 0, f^{(d+1)}\right) \\
& \leq T\left(r, f^{(d+1)}\right)+m T^{3 / 4}(r, f)+o(T(r, f)) \quad\left(r \rightarrow \infty, z=r e^{i \theta}\right) .
\end{aligned}
$$

Then

$$
\sigma(r) \leq \frac{1}{\mu_{j}}\left(T\left(r, f^{(d+1)}\right)+m T^{3 / 4}(r, f)+o(T(r, f))\right) \quad(r \rightarrow \infty) .
$$

From the lemma on logarithmic derivative (see: [16], p. 63) and (3.3), we have

$$
T\left(T_{j}, f^{(d+1)}\right) \leq O\left(\log T\left(S_{j}, f\right)\right)+T\left(T_{j}, f\right) \leq \frac{3}{2} T\left(S_{j}, f\right) \quad(j \rightarrow \infty) .
$$

Thus

$$
\sigma\left(T_{j}\right) \leq \frac{2}{\mu_{j}} T\left(S_{j}, f\right)
$$

Similarly, we have

$$
\sigma\left(S_{j}\right) \leq \frac{2}{\mu_{j}} T\left(R_{j}, f\right)
$$

From the monotonicity of $r \sigma^{\prime}(r)$ and Lemma $\mathrm{C}$ we get

$$
\sigma\left(S_{j}\right)-\sigma\left(T_{j}\right)=\int_{T_{j}}^{S_{j}} \sigma^{\prime}(r) d r \geq T_{j} \sigma^{\prime}\left(T_{j}\right) \log \frac{S_{j}}{T_{j}}=\frac{1}{\mu_{j}} T_{j} \sigma^{\prime}\left(T_{j}\right) .
$$


Hence

$$
T_{j} \sigma^{\prime}\left(T_{j}\right) \leq \mu_{j} \sigma\left(S_{j}\right) \leq 2 T\left(R_{j}, f\right) .
$$

From these inequalities and (3.4) we have

$$
\begin{aligned}
\left.\left(\frac{\sigma^{\prime}(r)}{r^{\mu_{j}-1}}+\mu_{j} \frac{\sigma(r)}{r^{\mu_{j}}}\right)\right|_{t_{j}} ^{T_{j}} & \leq \frac{4 T\left(R_{j}, f\right)}{R_{j}^{\mu_{j}}}-\frac{\sigma^{\prime}(1)}{t_{j}^{\mu_{j}}} \leq \frac{4 T\left(R_{j}, f\right)}{R_{j}^{\mu_{j}}}+\frac{T\left(t_{j}, f\right)}{t_{j}^{\mu_{j}}} \\
& <\varepsilon \mu_{j} \int_{t_{j}}^{T_{j}} \frac{T(r, f)}{r^{\mu_{j}+1}} d r \quad(j \rightarrow \infty) .
\end{aligned}
$$

Thus from (4.1) we have

$$
\begin{aligned}
& \frac{\sin \left(\mu_{j} \alpha\right)}{\pi} \sum_{k=1}^{m} \int_{t_{j}}^{T_{j}} \frac{\widetilde{u}_{j, k}(r, 0)}{r^{\mu_{j}+1}} d r-\mu_{j} \int_{t_{j}}^{T_{j}} \frac{T\left(r, f^{(d+1)}\right)}{r^{\mu_{j}+1}} d r+\mu_{j} \cos \left(\mu_{j} \alpha\right) \int_{t_{j}}^{T_{j}} \frac{N\left(r, 0, f^{(d+1)}\right)}{r^{\mu_{j}+1}} \\
& \quad<\varepsilon \mu_{j} \int_{t_{j}}^{T_{j}} \frac{T(r, f)}{r^{\mu_{j}+1}} d r \quad(j \rightarrow \infty) .
\end{aligned}
$$

Therefore we have

$$
\frac{\sin \left(\mu_{j} \alpha\right)}{\pi} \sum_{k=1}^{m} \int_{t_{j}}^{T_{j}} \frac{\widetilde{u}_{j, k}(r, 0)}{r^{\mu_{j}+1}} d r-\mu_{j} \int_{t_{j}}^{T_{j}} \frac{T\left(r, f^{d+1}\right)}{r^{\mu_{j}+1}} d r<\varepsilon \mu_{j} \int_{t_{j}}^{T_{j}} \frac{T(r, f)}{r^{\mu_{j}+1}} d r(j \rightarrow \infty) .
$$

It is clear that for meromorphic function $f$ with $(2.1)$

$$
\begin{aligned}
T\left(r, f^{(d+1)}\right) & =m\left(r, f^{(d+1)}\right)+N\left(r, f^{(d+1)}\right) \\
& \leq m\left(r, \frac{f^{(d+1)}}{f}\right)+T(r, f)+o(T(r, f)) \quad(r \rightarrow \infty) .
\end{aligned}
$$

Therefore Lemma A implies that

$$
\int_{t_{j}}^{T_{j}} \frac{T\left(r, f^{(d+1)}\right)}{r^{\mu_{j}+1}} d r<(1+\varepsilon) \int_{t_{j}}^{T_{j}} \frac{T(r, f)}{r^{\mu_{j}+1}} d r \quad(j \rightarrow \infty) .
$$

From this inequality and (4.3), we obtain

$$
\sum_{k=1}^{m} \int_{t_{j}}^{T_{j}} \frac{\widetilde{u}_{j, k}(r, 0)}{r^{\mu_{j}+1}} d r<\frac{(1+\varepsilon) \pi \mu_{j}}{\sin \left(\mu_{j} \alpha\right)} \int_{t_{j}}^{T_{j}} \frac{T(r, f)}{r^{\mu_{j}+1}} d r .
$$

Using (3.2) we have

(4.5) $\mu_{j} \int_{t_{j}}^{T_{j}} \frac{T(r, f)}{r^{\mu_{j}+1}} d r=\frac{T\left(t_{j}, f\right)}{t_{j}^{\mu_{j}}}-\frac{T\left(T_{j}, f\right)}{T_{j}^{\mu_{j}}}+\int_{t_{j}}^{T_{j}} \frac{r T_{-}^{\prime}(r, f)}{r^{\mu_{j}+1}} d r<(1+\varepsilon) \int_{t_{j}}^{T_{j}} \frac{A(r, f)}{r^{\mu_{j}+1}} d r$, $j \rightarrow \infty$. 
As $b\left(p_{k}, f\right)>0$, then

$$
\mathscr{L}\left(r, p_{k}, f\right)=\max _{|z|=r} \log ^{+} \frac{1}{\left|f(z)-p_{k}\right|}=\log ^{+} \frac{1}{\left|f\left(r e^{i \theta_{k}(r)}\right)-p_{k}\right|} \rightarrow \infty \quad(r \rightarrow \infty) .
$$

Thus for $j \rightarrow \infty$ and all $r \in\left[t_{j}, T_{j}\right]$ we have $\left|f\left(r e^{i \theta_{k}(r)}\right)-p_{k}\right|<\frac{c}{4}$. It is clear that

$$
\mathscr{L}\left(r, p_{k}, f\right) \leq \log ^{+}\left|\frac{f^{(d+1)}\left(r e^{i \theta_{k}(r)}\right)-p_{k}^{(d+1)}\left(r e^{i \theta_{k}(r)}\right)}{f\left(r e^{i \theta_{k}(r)}\right)-p_{k}\left(r e^{i \theta_{k}(r)}\right)}\right|+\log ^{+}\left|\frac{1}{f^{(d+1)}\left(r e^{i \theta_{k}(r)}\right)}\right| .
$$

Hence, if $\left|f^{(d+1)}\left(r e^{i \theta_{k}(r)}\right)\right| \geq \exp \left(-\sqrt{T\left(\rho_{j}, f\right)}\right)$, then

$$
\mathscr{L}\left(r, p_{k}, f\right) \leq \log ^{+}\left|\frac{f^{(d+1)}\left(r e^{i \theta_{k}(r)}\right)}{f\left(r e^{i \theta_{k}(r)}\right)-p_{k}\left(r e^{i \theta_{k}(r)}\right)}\right|+\sqrt{T\left(\rho_{j}, f\right)}
$$

If $\left|f^{(d+1)}\left(r e^{i \theta_{k}(r)}\right)\right|<\exp \left(-\sqrt{T\left(\rho_{j}, f\right)}\right)$, and

$$
\left|f^{(d)}\left(r e^{i \theta_{k}(r)}\right)-p_{k}^{(d)}\left(r e^{i \theta_{k}(r)}\right)\right| \geq \exp \left(-\sqrt{T\left(\rho_{j}, f\right)}\right),
$$

then

$$
\log ^{+} \frac{1}{\left|f\left(r e^{i \theta_{k}(r)}\right)-p_{k}\left(r e^{i \theta_{k}(r)}\right)\right|} \leq \log ^{+}\left|\frac{f^{(d)}\left(r e^{i \theta_{k}(r)}\right)-p^{(d)}\left(r e^{i \theta_{k}(r)}\right)}{f\left(r e^{i \theta_{k}(r)}\right)-p_{k}\left(r e^{i \theta_{k}(r)}\right)}\right|+\sqrt{T\left(\rho_{j}, f\right)} .
$$

If $\left|f^{(d+1)}\left(r e^{i \theta_{k}(r)}\right)\right|<\exp \left(-\sqrt{T\left(\rho_{j}, f\right)}\right)$,

$$
\begin{aligned}
\left|f^{(d)}\left(r e^{i \theta_{k}(r)}\right)-p_{k}^{(d)}\left(r e^{i \theta_{k}(r)}\right)\right| & <\exp \left(-\sqrt{T\left(\rho_{j}, f\right)}\right), \ldots,\left|f^{\prime \prime}\left(r e^{i \theta_{k}(r)}\right)-p_{k}^{\prime \prime}\left(r e^{i \theta_{k}(r)}\right)\right| \\
& <\exp \left(-\sqrt{T\left(\rho_{j}, f\right)}\right)
\end{aligned}
$$

and

$$
\left|f^{\prime}\left(r e^{i \theta_{k}(r)}\right)-p_{k}^{\prime}\left(r e^{i \theta_{k}(r)}\right)\right| \geq \exp \left(-\sqrt{T\left(\rho_{j}, f\right)}\right)
$$

then

$$
\log ^{+} \frac{1}{\left|f\left(r e^{i \theta_{k}(r)}\right)-p_{k}\left(r e^{i \theta_{k}(r)}\right)\right|} \leq \log ^{+}\left|\frac{f^{\prime}\left(r e^{i \theta_{k}(r)}\right)-p^{\prime}\left(r e^{i \theta_{k}(r)}\right)}{f\left(r e^{i \theta_{k}(r)}\right)-p_{k}\left(r e^{i \theta_{k}(r)}\right)}\right|+\sqrt{T\left(\rho_{j}, f\right)} .
$$

If $\left|f^{(l)}\left(r e^{i \theta_{k}(r)}\right)-p_{k}^{(l)}\left(r e^{i \theta_{k}(r)}\right)\right|<\exp \left(-\sqrt{T\left(\rho_{j}, f\right)}\right)$, where $l=1,2, \ldots, d+1$, then

$$
\log ^{+}\left|\frac{1}{f^{(d+1)}\left(r e^{i \theta_{k}(r)}\right)}\right| \leq \widetilde{u}_{j, k}(r)
$$

Thus,

$$
\begin{aligned}
\log ^{+} & \frac{1}{\mid f\left(r e^{\left.i \theta_{k}(r)\right)}-p_{k}\left(r e^{i \theta_{k}(r)}\right) \mid\right.} \leq \widetilde{u}_{j, k}(r)+\log ^{+} M\left(r, \frac{\left(f-p_{k}\right)^{\prime}}{f-p_{k}}\right) \\
& +\log ^{+} M\left(r, \frac{\left(f-p_{k}\right)^{\prime \prime}}{f-p_{k}}\right)+\ldots+\log ^{+} M\left(r, \frac{\left(f-p_{k}\right)^{(d+1)}}{f-p_{k}}\right)+\sqrt{T\left(\rho_{j}, f\right)},
\end{aligned}
$$

$1 \leq k \leq m$. From this and (3.5) we have, that for all $r \in\left[t_{j}, T_{j}\right]$

$$
\sum_{k=1}^{m} \mathscr{L}\left(r, p_{k}, f\right) \leq \sum_{k=1}^{m} \widetilde{u}_{j, k}(r)+\sum_{k=1}^{m} \sum_{i=1}^{d+1} \log ^{+} M\left(r, \frac{\left(f-p_{k}\right)^{(i)}}{f-p_{k}}\right)+m T^{3 / 4}(r, f)
$$


Using (4.5), (4.6) and Lemma B we have that for $j \geq j_{0}(\varepsilon)$

$$
\sum_{k=1}^{m} \int_{t_{j}}^{T_{j}} \frac{\mathscr{L}\left(r, p_{k}, f\right)}{r^{\mu_{j}+1}} d r<\frac{(1+\varepsilon) \pi}{\sin \left(\mu_{j} \alpha\right)} \int_{t_{j}}^{T_{j}} \frac{A(r, f)}{r^{\mu_{j}+1}} d r .
$$

Passing to the limit with $\alpha \rightarrow \frac{\pi}{2 \mu_{j}}$ we obtain

$$
\sum_{k=1}^{m} \int_{t_{j}}^{T_{j}} \frac{\mathscr{L}\left(r, p_{k}, f\right)}{r^{\mu_{j}+1}} d r<(1+\varepsilon) \pi \int_{t_{j}}^{T_{j}} \frac{A(r, f)}{r^{\mu_{j}+1}} d r \quad(j \rightarrow \infty) .
$$

Therefore there exists sequence $r_{j} \in\left[t_{j}, T_{j}\right]$ such that

$$
\sum_{k=1}^{q} \mathscr{L}\left(r_{j}, p_{k}, f\right)<(1+\varepsilon) \pi A\left(r_{j}, f\right) \quad(j \rightarrow \infty) .
$$

Hence

$$
\sum_{k=1}^{m} b\left(p_{k}, f\right) \leq(1+\varepsilon) \pi
$$

As $\varepsilon>0$ was chosen arbitrarily, we have

$$
\sum_{k=1}^{m} b\left(p_{k}, f\right) \leq \pi
$$

Thus we obtain the statement for polynomials.

Let now $\left\{q_{k}(z)\right\}_{k=1}^{m}$ be a set of distinct rational functions. Let $q_{k}(z)=\frac{P_{k}(z)}{Q_{k}(z)}$, where $P_{k}(z), Q_{k}(z)$ are polynomials, $k=1,2, \ldots, m$. We put

$$
p(z)=\prod_{k=1}^{m} Q_{k}(z), \quad F(z)=f(z) p(z), \quad p_{k}(z)=p(z) q_{k}(z) .
$$

It is clear that

$$
\begin{aligned}
\sum_{k=1}^{m} b\left(q_{k}, f\right) & =\sum_{k=1}^{m} \liminf _{r \rightarrow \infty} \frac{\mathscr{L}\left(r, q_{k}, f\right)}{A(r, f)}=\sum_{k=1}^{m} \liminf _{r \rightarrow \infty} \frac{\max _{|z|=r} \log ^{+} \frac{1}{\left|f(z)-q_{k}\right|}}{A(r, f)} \\
& =\sum_{k=1}^{m} \liminf _{r \rightarrow \infty} \frac{\max _{|z|=r} \log ^{+} \frac{p(z)}{\left|F(z)-p_{k}(z)\right|}}{A(r, f)} \\
& \leq \sum_{k=1}^{m} \liminf _{r \rightarrow \infty} \frac{\max _{|z|=r} \log ^{+} \frac{1}{\left|F(z)-p_{k}(z)\right|}}{A(r, f)}+\limsup _{r \rightarrow \infty} m \frac{\log ^{+} \max _{|z|=r}|p(z)|}{A(r, f)}
\end{aligned}
$$

As $f(z)$ is an entire function of infinite lower order, then $A(r, f)$ is also a function of infinite lower order. Therefore

$$
\log ^{+} M(r, p)=O(\log r)=o(A(r, f)) \quad(r \rightarrow \infty) .
$$

Hence

$$
\sum_{k=1}^{m} b\left(q_{k}, f\right) \leq \sum_{k=1}^{m} b\left(p_{k}, F\right) \leq \pi
$$


Let

$$
\Omega=\{q \in \mathfrak{M}: b(q, f)>0\}, \quad \Omega_{n}=\left\{q \in \mathfrak{M}: b(q, f)>\frac{1}{n}\right\}, \quad n \in \mathbf{N} .
$$

It is clear that

$$
\Omega=\bigcup_{n=1}^{\infty} \Omega_{n} .
$$

From (4.7) we have, that for each $n \in \mathbf{N}$ the set $\Omega_{n}$ is finite. Then the set $\Omega$ is at most countable. Thus the statement of Theorem 1 for meromorphic functions infinite lower order we get from (4.7).

Proof of Theorem 1 for meromorphic functions of finite lower order $\lambda, 0<\lambda<\infty$, is similar to the given proof, need to use the usual peaks Polya and $\mu_{j}=\lambda$ (see also [24]).

\section{Proof of Theorem 2}

First we shall prove the statement for meromorphic functions $f(z)$ of infinite lower order. As in the proof of Theorem 1 from ([17], p. 30) we obtain, that for all $r \in\left[t_{j}, T_{j}\right]$

$$
\begin{aligned}
r \frac{d}{d r} r \sigma^{\prime}(r) \geq & \frac{1}{\pi} \sum_{k=1}^{m} \widetilde{u}_{j, k}(r, 0) \sin \left(\mu_{j} \alpha\right)-\mu_{j} T_{0}^{*}\left(r e^{i \alpha}, f\right) \\
& +\mu_{j} \cos \left(\mu_{j} \alpha\right) N\left(r, 0, f^{(d+1)}\right)+\mu_{j}^{2} \sigma(r):=h(r)+\mu_{j}^{2} \sigma(r) .
\end{aligned}
$$

We divide this inequality by $r^{\mu_{j}+1}$ and integrate it over the interval $\left[t_{j}, T_{j}\right]$ applying integration by parts and Lemma C. We get

$$
\int_{t_{j}}^{T_{j}} \frac{h(r)}{r^{\mu_{j}+1}} d r \leq\left.\left(\frac{\sigma(r)}{r^{\mu_{j}-1}}+\mu_{j} \frac{\sigma(r)}{r^{\mu_{j}}}\right)\right|_{t_{j}} ^{T_{j}}
$$

It follows from the definition of function $T_{0}^{*}(z, f)$ and inequality (3.4) that for all $\theta \in[0, \pi], r \in\left[t_{j}, T_{j}\right]$

$$
\begin{aligned}
T_{0}^{*}(z, f) & \leq m\left(r, 0, f^{(d+1)}\right)+m \sqrt{T\left(\rho_{j}, f\right)}+N\left(r, 0, f^{(d+1)}\right) \\
& \leq T\left(r, f^{(d+1)}\right)+m T^{3 / 4}(r, f) \quad\left(z=r e^{i \theta}\right) .
\end{aligned}
$$

Then

$$
\sigma(r) \leq \frac{1}{\mu_{j}}\left(T\left(r, f^{(d+1)}\right)+m T^{3 / 4}(r, f)\right) .
$$

From the lemma on logarithmic derivative (see: [16], p. 63)) and (3.3), we have

$$
\begin{aligned}
T\left(T_{j}, f^{(d+1)}\right) & \leq O\left(\log T\left(S_{j}, f\right)\right)+T\left(T_{j}, f\right)+N\left(T_{j}, f^{(d+1)}\right) \\
& \leq\left(d+2+\frac{1}{2}\right) T\left(S_{j}, f\right) \quad(j \rightarrow \infty) .
\end{aligned}
$$

Thus

$$
\sigma\left(T_{j}\right) \leq \frac{d+3}{\mu_{j}} T\left(S_{j}, f\right) .
$$


Similarly, we have

$$
\sigma\left(S_{j}\right) \leq \frac{d+3}{\mu_{j}} T\left(R_{j}, f\right) .
$$

From the monotonicity of $r \sigma^{\prime}(r)$, we get

$$
\sigma\left(S_{j}\right)-\sigma\left(T_{j}\right)=\int_{T_{j}}^{S_{j}} \sigma^{\prime}(r) d r \geq T_{j} \sigma^{\prime}\left(T_{j}\right) \log \frac{S_{j}}{T_{j}}=\frac{1}{\mu_{j}} T_{j} \sigma^{\prime}\left(T_{j}\right) .
$$

Hence

$$
T_{j} \sigma^{\prime}\left(T_{j}\right) \leq \mu_{j} \sigma\left(S_{j}\right) \leq(d+3) T\left(R_{j}, f\right) .
$$

From these inequalities and (3.4), we have

$$
\begin{aligned}
\left.\left(\frac{\sigma^{\prime}(r)}{r^{\mu_{j}-1}}+\mu_{j} \frac{\sigma(r)}{r^{\mu_{j}}}\right)\right|_{t_{j}} ^{T_{j}} & \leq \frac{(d+3) e^{2} T\left(R_{j}, f\right)}{R_{j}^{\mu_{j}}}-\frac{\sigma^{\prime}(1)}{t_{j}^{\mu_{j}}} \leq \frac{(d+3) e^{2} T\left(R_{j}, f\right)}{R_{j}^{\mu_{j}}}+\frac{T\left(t_{j}, f\right)}{t_{j}^{\mu_{j}}} \\
& <\varepsilon \mu_{j} \int_{t_{j}}^{T_{j}} \frac{T(r, f)}{r^{\mu_{j}+1}} d r \quad(j \rightarrow \infty) .
\end{aligned}
$$

Thus from (5.1) we have

$$
\frac{\sin \left(\mu_{j} \alpha\right)}{\pi} \sum_{k=1}^{m} \int_{t_{j}}^{T_{j}} \frac{\widetilde{u}_{j, k}(r, 0)}{r^{\mu_{j}+1}} d r-\mu_{j} \int_{t_{j}}^{T_{j}} \frac{T\left(r, f^{(d+1)}\right)}{r^{\mu_{j}+1}} d r+\mu_{j} \cos \left(\mu_{j} \alpha\right) \int_{t_{j}}^{T_{j}} \frac{N\left(r, 0, f^{(d+1)}\right)}{r^{\mu_{j}+1}}
$$

$$
<\varepsilon \mu_{j} \int_{t_{j}}^{T_{j}} \frac{T(r, f)}{r^{\mu_{j}+1}} d r \quad(j \rightarrow \infty) .
$$

Let us recall the definition of Valiron's deficiency:

$$
\Delta\left(0, f^{(d+1)}\right):=\limsup _{r \rightarrow \infty}\left(1-\frac{N\left(r, 0, f^{(d+1)}\right)}{T\left(r, f^{(d+1)}\right)}\right) .
$$

Hence for $r \geq r_{0}(\varepsilon)$ we have

$$
N\left(r, 0, f^{(d+1)}\right)>\left(1-\Delta\left(0, f^{(d+1)}\right)-\varepsilon\right) T\left(r, f^{(d+1)}\right),
$$

where $\varepsilon>0$ is arbitrary fixed number. It is clear that

$$
T\left(r, f^{(d+1)}\right)=m\left(r, f^{(d+1)}\right)+N\left(r, f^{(d+1)}\right) \leq m\left(r, \frac{f^{(d+1)}}{f}\right)+(d+2) T(r, f) .
$$

Therefore Lemma A implies that

$$
\int_{t_{j}}^{T_{j}} \frac{T\left(r, f^{(d+1)}\right)}{r^{\mu_{j}+1}} d r<(d+2+\varepsilon) \int_{t_{j}}^{T_{j}} \frac{T(r, f)}{r^{\mu_{j}+1}} d r \quad(j \rightarrow \infty) .
$$

From this inequality, (5.2) and (5.3) we obtain

$$
\sum_{k=1}^{m} \int_{t_{j}}^{T_{j}} \frac{\widetilde{u}_{j, k}(r, 0)}{r^{\mu_{j}+1}} d r<\frac{(d+2+\varepsilon) \pi \mu_{j}}{\sin \left(\mu_{j} \alpha\right)}\left(1-(1-\Delta) \cos \left(\mu_{j} \alpha\right)+\varepsilon\right) \int_{t_{j}}^{T_{j}} \frac{T(r, f)}{r^{\mu_{j}+1}} d r
$$


where $\Delta=\Delta\left(0, f^{d+1}\right)$. From (4.6), (5.4) and Lemma B we have that for $j \geq j_{0}(\varepsilon)$

$$
\sum_{k=1}^{m} \int_{t_{j}}^{T_{j}} \frac{\mathscr{L}\left(r, p_{k}, f\right)}{r^{\mu_{j}+1}} d r<\frac{(d+2+\varepsilon)\left(1-(1-\Delta) \cos \left(\mu_{j} \alpha\right)+\varepsilon\right) \pi}{\sin \left(\mu_{j} \alpha\right)}(1+\varepsilon) \int_{t_{j}}^{T_{j}} \frac{A(r, f)}{r^{\mu_{j}+1}} d r .
$$

Next we let $\alpha=\frac{1}{\mu_{j}} \arccos (1-\Delta)$. Then

$$
\sum_{k=1}^{m} \int_{t_{j}}^{T_{j}} \frac{\mathscr{L}\left(r, p_{k}, f\right)}{r^{\mu_{j}+1}} d r<(d+2+\varepsilon) \pi(\sqrt{\Delta(2-\Delta)}+\varepsilon)(1+\varepsilon) \int_{t_{j}}^{T_{j}} \frac{A(r, f)}{r^{\mu_{j}+1}} d r \quad(j \rightarrow \infty) .
$$

Therefore there exists the sequence $r_{j} \in\left[t_{j}, T_{j}\right]$ such that

$$
\sum_{k=1}^{m} \mathscr{L}\left(r_{j}, p_{k}, f\right)<(d+2+\varepsilon) \pi(\sqrt{\Delta(2-\Delta)}+\varepsilon)(1+\varepsilon) A\left(r_{j}, f\right) \quad(j \rightarrow \infty) .
$$

Hence

$$
\sum_{k=1}^{m} b\left(p_{k}, f\right) \leq(d+2+\varepsilon) \pi(\sqrt{\Delta(2-\Delta)}+\varepsilon)(1+\varepsilon) .
$$

As $\varepsilon>0$ was chosen arbitrarily, we have

$$
\sum_{k=1}^{m} b\left(p_{k}, f\right) \leq(d+2) \sqrt{\Delta(2-\Delta)} \pi .
$$

Thus the statement of Theorem 2 for meromorphic functions of infinite lower order we get from (5.5).

Proof of Theorem 2 for meromorphic functions of finite lower order $\lambda, 0<\lambda<\infty$, is similar to the given proof, need to use the usual peaks Polya and $\mu_{j}=\lambda$ (see also [21]).

\section{References}

[1] Baernstein, A.: Integral means, univalent functions and circular symmetrization. - Acta Math. 133:3-4, 1974, 139-169.

[2] Bergweiler, W., and H. Bock: On the growth of meromorphic functions of infinite order. - J. Anal. Math. 64, 1994, 327-336.

[3] Ciechanowicz, E., and I. I. Marchenko: On deviations from rational functions of entire functions of finite lower order. - Ann. Polon. Math. 91:2-3, 2007, 161-177.

[4] Eremenko, A. E.: The inverse problem of the theory of value distribution of finite lower order meromorphic functions. - Sib. Math. J. 3:27, 1986, 87-102 (in Russian); English transl.: Sib. Math. J. 27, 1986, 377-390.

[5] Eremenko, A.: An analogue of the defect relation for the uniform metric. - Complex Var. Theory Appl. 34, 1997, 83-97.

[6] Essen, M., and D.F. SheA: Applications of Denjoy integral inequalities and differential inequalities to growth problems for subharmonic and meromorphic functions. - Proc. Roy. Irish Acad. A82, 1982, 201-216.

[7] Fenton, P. C.: Entire functions having asymptotic functions. - Bull. Austral. Math. Soc. 27, $1983,321-328$.

[8] Frank, G., and G. Weissenborn: Rational deficient functions of meromorphic functions. Bull. London Math. Soc. 18, 1986, 29-33. 
[9] Fuchs, W. H. J.: Topics in Nevanlinna theory. - In: Proceedings of the NRL Conference on Classical Function Theory, Math. Res. Center, Naval Res. Lab., Washington, D.C., 1970, 1-32.

[10] Gariepy, R., and J.L. Lewis: Space analogues of some theorems for subharmonic and meromorphic functions. - Ark. Mat. 13, 1975, 91-105.

[11] Gol'dberg, A. A., and I. V. Ostrowskir: Some theorems on the growth of meromorphic functions. - Zap. Mat. Otd. Kharkov University and Kharkov Math. Soc. 4:27, 1961, 3-37 (in Russian).

[12] Gol'dberg, A. A., and I. V. Ostrowskir: Value distribution of meromorphic functions. Nauka, Moscow, 1970 (in Russian); English transl.: Transl. Math. Monogr. 236, Amer. Math. Soc., Providance, 2008.

[13] Govorov, N. V.: On Paley's problem. - Funct. Anal. Appl. 3:2, 1969, 38-43 (in Russian).

[14] Gross, W.: Eine ganze Function für die jede komplexe Zahl Konvergenzwert ist. - Math. Ann. 79, 1918, 201-208.

[15] Hayman, W. K.: Multivalent Functions. - Cambridge Univ. Press, Cambridge, 1958.

[16] Hayman, W. K.: Meromorphic functions. - Oxford, Clarendon Press, 1964.

[17] Kaluzhynova, L. V., and I. I. Marchenko: On the magnitudes of deviations of entire functions of infinite order from rational functions. - Mat. Zametki 85:1, 2009, 22-35 (in Russian); English transl.: Math. Notes 85:1, 2009, 20-33.

[18] LAIne, I.: Nevanlinna theory and complex differential equations. - de Gruyter, Berlin, 1993.

[19] Marchenko, I. I.: On the magnitudes of deviations and spreads of meromorphic functions of finite lower order. - Mat. Sb. 186, 1995, 85-102 (in Russian); English transl.: Sb. Math. 186, $1995,391-408$.

[20] Marchenko, I. I.: On the growth of entire and meromorphic functions. - Mat. Sb. 189:6, 1998, 59-84 (in Russian); English transl.: Sb. Math. 189, 1998, 875-900.

[21] Marchenko, I. I.: On the devitations and defects of meromorphic functions of finite lower order. - Ukraïn. Mat. Zh. 51:6, 1999, 784-791 (in Russian); English transl.: Ukrainian Math. J. 51:6, 1999, 796-803.

[22] Marchenko, I. I.: On the strong asymptotic spots of meromorphic functions of finite lower order. - Mat. Fiz. Anal. Geom. 11, 2004, 484-491 (in Russian).

[23] Marchenko, I. I.: On the growth of entire and meromorphic functions. - Mat. Stud. 30:2, 2008, 139-146.

[24] Marchenko, I. I. and A. I. Shcherba: On the magnitudes of deviations of meromorphic functions. - Mat. Sb. 181:1, 1990, 3-24 (in Russian); English transl.: Sb. Math. 69:1, 1991, $1-24$.

[25] Natanson, E. P.: The theory of functions of a real variable. - Revised edition, Ungar, New York, 1961.

[26] Nevanlinna, R.: Analytic functions. - Revised transl. by P. Emig of Eindeutige analytische Functionen, 2nd edition, Springer, Berlin, 1970.

[27] Paley, R.: A note of integral function. - Proc. Cambridge Philos. Soc. 28, 1932, 262-265.

[28] Petrenko, V. P.: The growth of meromorphic functions of finite lower order. - Izv. Akad. Nauk USSR 33:2, 1969, 414-454 (in Russian).

[29] Petrenko, V.P.: Growth of meromorphic functions. - Vischa Shkola, Kharkov, 1978 (in Russian).

[30] Ryzhkov, A. M.: On the exactness of the estimate of the value of deviation for a meromorphic function. - Teor. Funktsiǔ Funktsional. Anal. i Prilozhen. 37, 1982, 114-116 (in Russian).

[31] Steinmetz, N.: Eine Verallgemainerung des zweiten Nevanlinnaschen Hauptsatzes. - J. Reine Angew. Math. 368, 1986, 134-141.

Received 30 December 2010 